\title{
Imaging Relativistic Radio Jets and the X-ray-Radio Connection In X-ray Transients
}

\author{
R. M. Hjellming \\ National Radio Astronomy Observatory, Socorro, NM 87801
}

\begin{abstract}
The superluminal radio jets produced by GRO J1655-40 during a series of X-ray outbursts in 1994 were the first indications of a delayed form of relativistic outflows from black hole accretion disk environments. In this paper we review the relation between the radio jets and changes in the X-ray environment for the 1994 events in GRO J165540 and compare this behavior with similar X-ray-radio correlations that have since been found in GRS 1716-249, GRS 1739-278, and the 1996 recurrence of another correlated event in GRO 1655-40. We also discuss newly found radio-X-ray correlations in a galactic X-ray binary, Cyg X-3.
\end{abstract}

\section{Introduction}

The ejection of relativistic jets, at a speed of $0.92 c$, in the galactic X-ray transients GRS1915+105 and GRO J1655-40, and the way this jet ejection is related to changes in the X-ray and radio light curves in GRO J1655-40, implies that there is a specific relationship, or connection, between the radio jets and prior peaks (or sudden decays) in the hard X-ray emission. In this review we summarize the X-ray-radio connection for GRO J1655-40 as shown in the events in 1994. We also discuss the radio quiet behavior of GRO J1655-40 during hard X-ray flaring in 1995 , and show the early comparison of $2-12 \mathrm{keV}, 20-100 \mathrm{keV}$, and radio flaring in GRO J1655-40's long-lasting event in 1996. The X-ray-radio connection for events in the X-ray transients GRS 1716-249 and GRS 1739-278 is also discussed. The data for the three $\mathrm{X}$-ray transients, for which we have simultaneous hard X-ray and radio light curves, indicates that the delay between a peak in the hard X-ray flux and the beginning of jet ejection is a few days or less, while the peaks in radio light curves occur tens of days later. We also summarize the hard X-ray-radio correlation during pre-flare and initial flare active for Cyg X-3 and the anti-correlation seen during periods of slowly varying, quiescent hard X-ray and radio emission; this behavior is present during the entire period for which BATSE and Green Bank Interferometer data for Cyg $\mathrm{X}-3$ is available.

\section{GRO J1655-40 in 1994 and 1995}

Between August and December 1994 the X-ray transient and black hole binary system GRO J1655-40 underwent a series of high energy X-ray flares, as seen by BATSE (Harmon et al. 1995), and radio flares observed and imaged with 


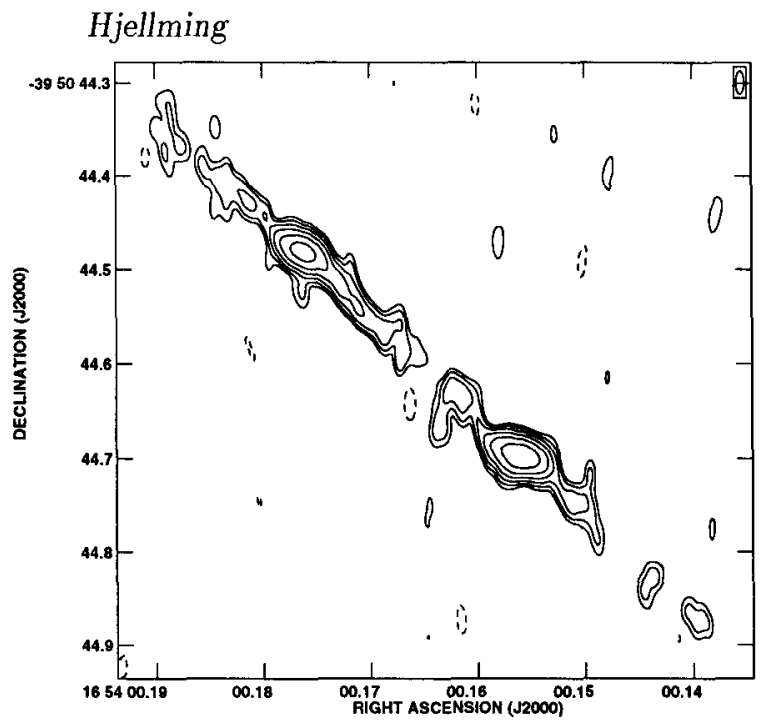

Figure 1. A high resolution image of the radio jets of GRO J165540 made from Aug. 18-19, 1994 VLBA data at $18 \mathrm{~cm}$. The contours correspond to $0.004(-1,1,2,4,8,16,32,64,128) \mathrm{Jy}$. The beam in the upper right corner is 25 by 9 milliarcseconds in size.

SHEVE (Tingay et al. 1995), VLBA, and VLA (Hjellming and Rupen 1995). In Figure 1 we show a high resolution $18 \mathrm{~cm}$ image of the Aug. 18-19, 1994 VLBA data which indicates the complex structure near the source of the $0.92 \mathrm{c}$ ejecta and the extended jets. The central structure changes significantly in subsequent VLBA images, indicating that there is nearly symmetrical ejection of synchrotron-radiating plasma at much wider angles from the jet axis than the $\pm 2^{\circ}$ cone angle of the outer jets, with transverse motions of ejected material.

As discussed by Harmon et al. (1995) and Hjellming and Rupen (1995) the BATSE X-ray flux at 2-100 keV had three peaks in 1994 that were followed by radio peaks roughly 13,2 , and 1 days later; however, the time delay between the beginning of ejection of jet segments is roughly $5,-2$, and 6 days, respectively. The jet ejections therefore began very close to the hard X-ray peaks. Longer delays between X-ray and radio peaks, as seen in Figure 2, are a result of the radio peak being determined by the compromise between the growth in integrated surface brightness, due to the increase in solid angle of the jets until ejection stops, and the decay that begins as soon as jet material is ejected.

Figure 2 also shows that while GRO J1655-40 had two major hard X-ray flares in 1995, it was radio quiet during those flares. Also indicated in Figure 2 are two days when there was Hubble Space Telescope (HST) high resolution optical imaging. No extended optical emission was observed, which probably correlates with the lack of radio jets at that time (Tavani et al. 1996).

\section{The 1996 X-ray-Radio Event in GRO J1655-40}

After more than a year of radio-quiet behavior, in early 1966 GRO J1655-40 underwent soft and hard X-ray flaring that was accompanied by sporadic radio 
The $X$-ray-Radio Connection In $X$-ray Transients
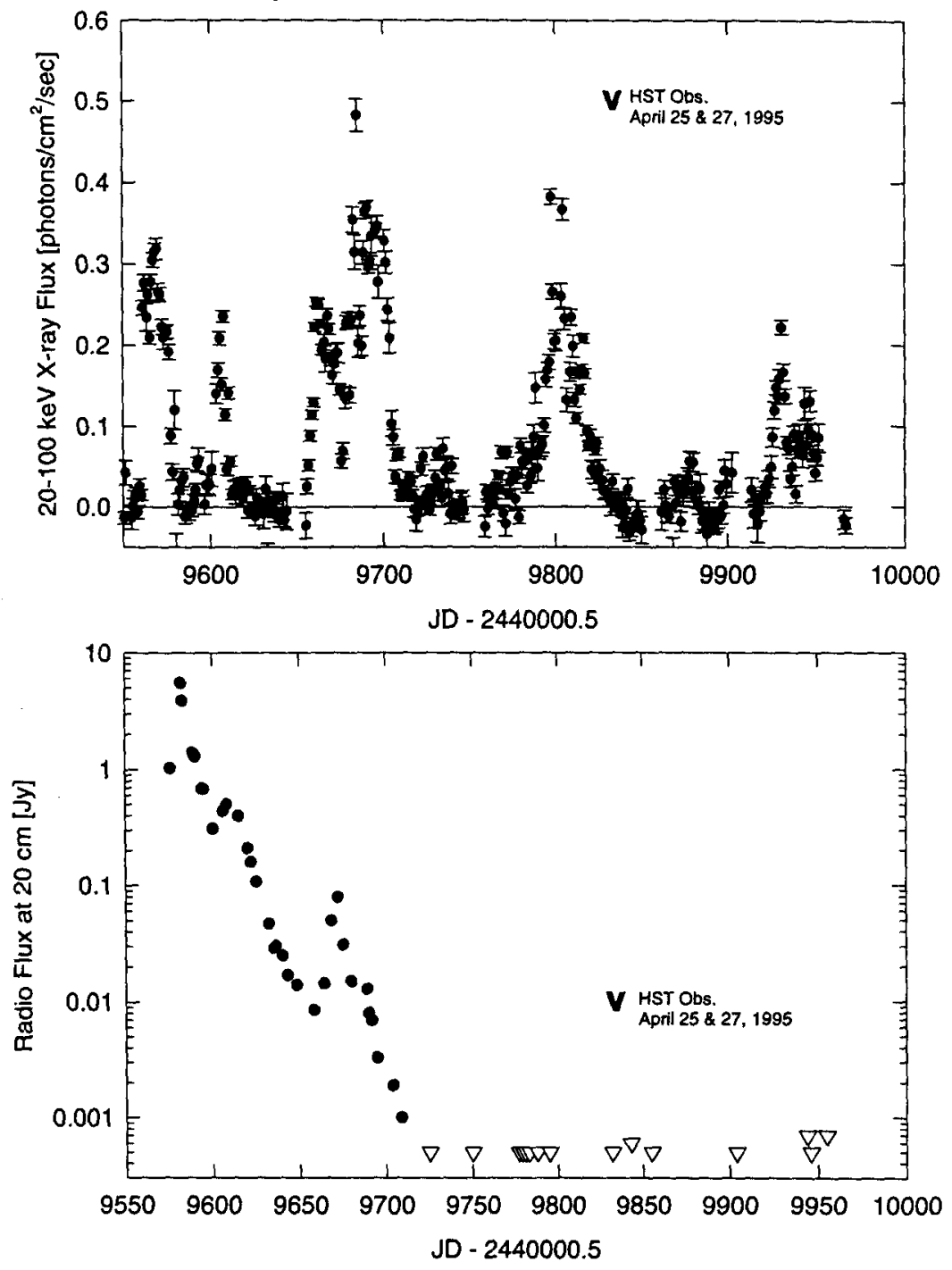

Figure 2. BATSE $20-100 \mathrm{keV}$ fluxes (top) and $20 \mathrm{~cm} \mathrm{VLA}$ radio fluxes (bottom) are plotted as a function of Julian Day (JD) -2440000.5 for the 1994-1995 flaring events in GRO J1655-40. The open triangles are VLA upper limits at 4.9 or $8.4 \mathrm{GHz}$. 

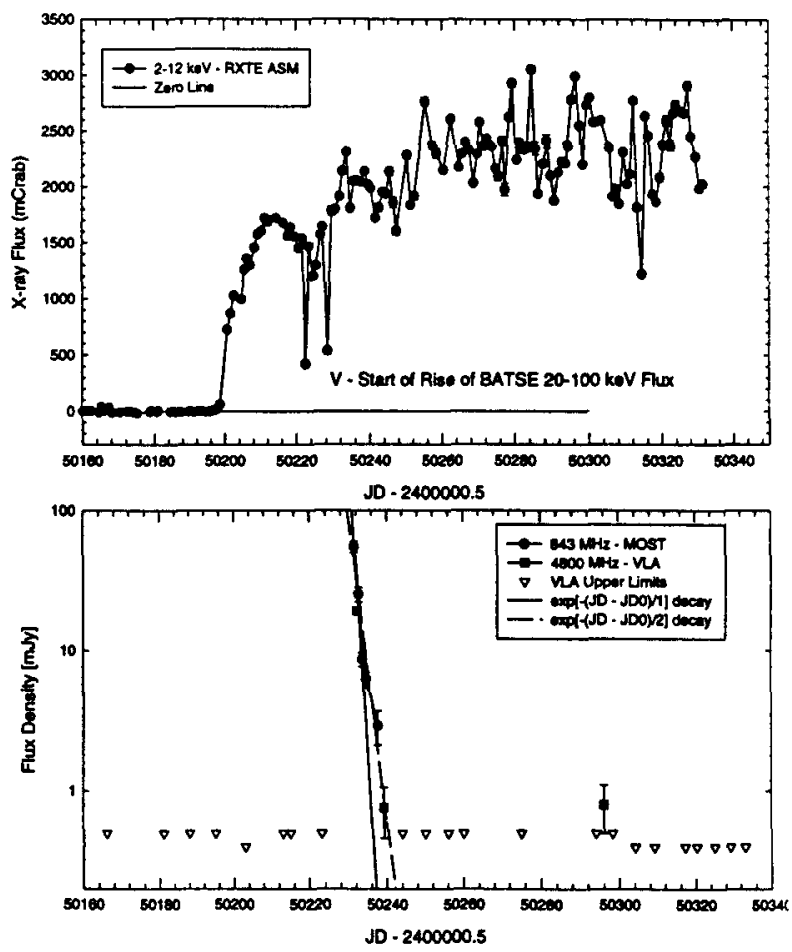

Figure 3. RXTE ASM 2-12 keV fluxes (filled circles,top) and BATSE $20-100 \mathrm{keV}$ fluxes (open diamonds, top), $843 \mathrm{GHz}$ MOST radio fluxes (filled circles, bottom), $4.9 \mathrm{GHZ}$ VLA radio fluxes (filled squares, bottom), and VLA radio upper limits (open triangles, bottom) are plotted as a function of Julian Day (JD) -2440000.5 for the 1996 flaring event in GRO J1655-40.

flares. As seen in Figure 3, a rise to a very long-lasting soft X-ray event began on JD 2450206. However, BATSE did not detect significant X-ray flux until JD 2450230 , and it was on JD 2450231 that the Molonglo Observatory Synthesis Telescope (MOST) detected a $55 \mathrm{mJy}$ radio source that decayed with an initial 1 day e-folding time. Further MOST and VLA observations showed that the entire event had an e-folding time of about 2 days. The VLA upper limits before and during the X-ray event define the peak of the radio flare as in the time range JD 2450223-2450231. This radio flare began 17-25 days after the start of the soft X-ray flare and very close to the beginning of hard X-ray emission. In addition, there was a VLA detection at $0.8 \mathrm{mJy}$ on JD 2450296, although it was undetectable two days before and two days afterwards.

\section{A $1995 \mathrm{X}$-ray-Radio Event in GRS 1716-249}

The X-ray transient GRS 1716-249 was first seen as X-ray Nova Ophiuchi 1993 (Ballet et al. 1993, Harmon et al. 1993). The optical and radio counterparts were 


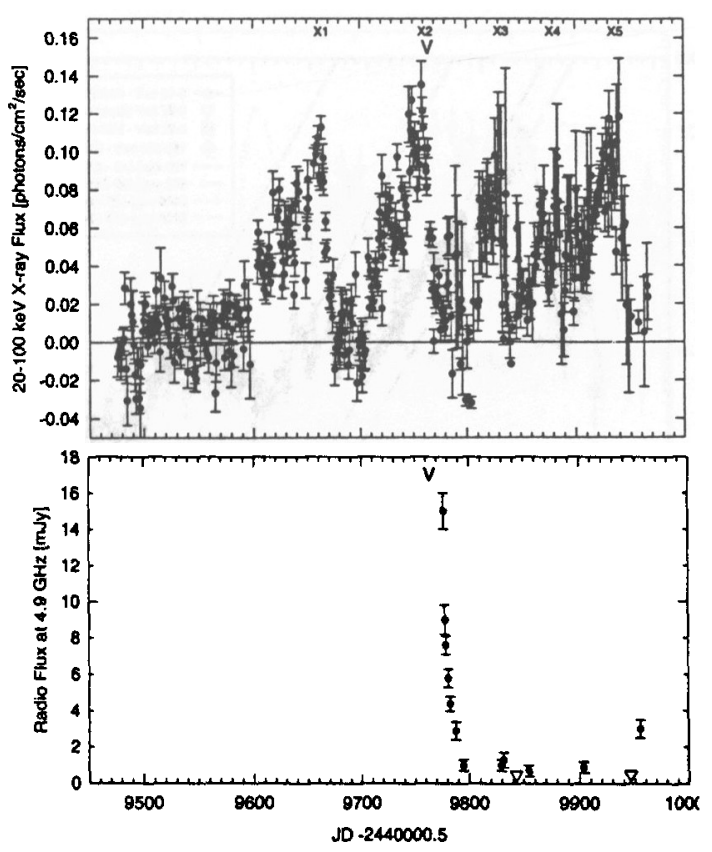

Figure 4. BATSE $20-100 \mathrm{keV}$ fluxes (top) and $4.9 \mathrm{GHz}$ VLA radio fluxes (bottom) are plotted as a function of Julian Day (JD) -2440000.5 for the 1996 flaring event in GRS 1716-249. The $\mathrm{V}$ at the top of each plot at 9764 marks the time MIR/Kvant observed that the X-ray source was active again.

detected by Della Valle, Mirabel, and Cordier (1993) and Mirabel, Rodriguez, and Cordier (1993). On 1995 February 16 the MIR/Kvant team (Borozdin et al. 1995) detected renewed strong X-ray emission. Shortly thereafter there were radio observations, by the MOST and the VLA, of a rapidly decaying radio flare as plotted in Figure 4. Subsequently a 2-100 keV BATSE light curve was obtained from the BATSE data archive, as shown in Figure 4. The strong radio flare behaved like the decay of an expanding, spherically symmetric, synchrotron radio source with an origin at about the time of the MIR/Kvant detection and the peak of the second (X2) of a series of five unusual "sawtooth"-like hard X-ray events (Hjellming et al. 1996). In addition, there were subsequent but weaker radio events that roughly matched the continued hard X-ray events, although only the last radio "flare" and the fifth (X5) hard X-ray peak can be even roughly associated.

\section{X-Ray-Radio Correlations in GRS 1739-278}

The X-ray transient GRS $1739-278$ was first reported as an X-ray source with a hard X-ray component by the SIGMA/Granat team (Paul et al. 1196) and was later found in MIR/Kvant 2-27 keV data (Borozdin et al. 1996) to have been undetected on JD 2450120, but was detected rising from 0.2 Crab to 0.5 


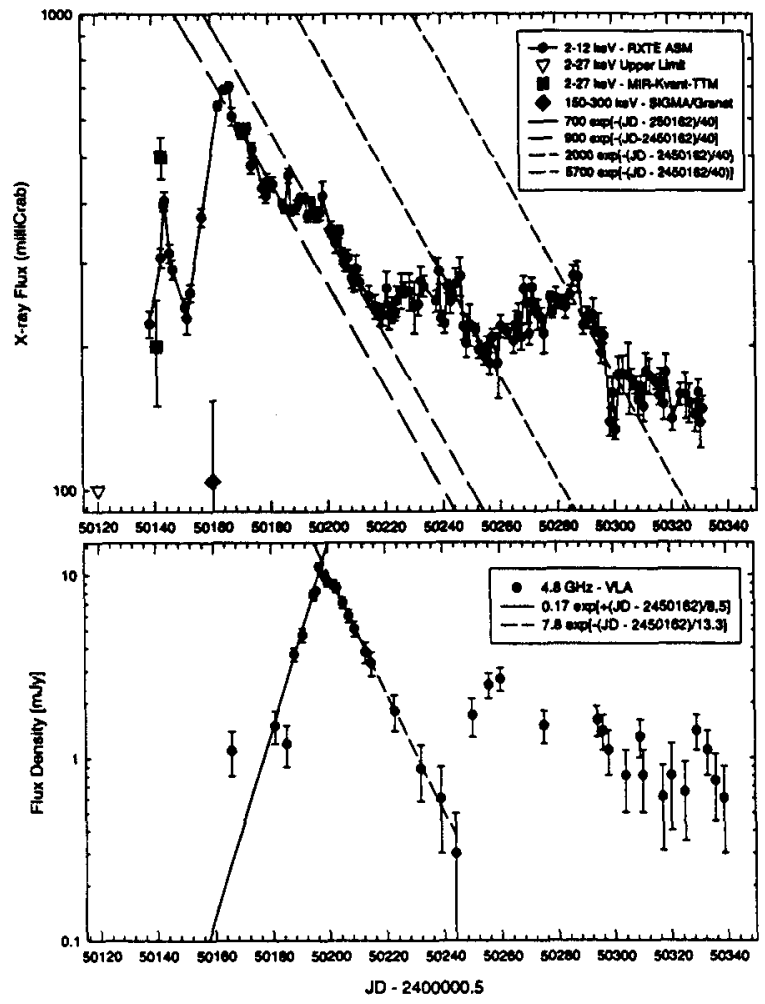

Figure 5. RXTE ASM 2-12 keV fluxes (top) and $4.9 \mathrm{GHz}$ VLA radio fluxes (bottom) are plotted as a function of Julian Day (JD) -2440000.5 for the 1995 flaring events in GRS 1739-278.

Crab between JD 2450141 and 2450143. As seen in the top panel in Figure 5, a mixture of extensive RXTE ASM data, MIR/Kvant data, and a detection by SIGMA/Granat on JD 2450160 indicats that there was strong precursor activity before the soft X-ray peak on JD 2450166 (Hjellming et al. 1997). Following the peak there was an exponential decay with an e-folding time of 40 days, which was interrupted by a series of "glitches" after which it returned to the same exponential decay. As shown in the bottom panel in Figure 5, a very well defined radio event with an exponential rise and decay, with e-folding times of 8.5 and 13.3 days, respectively, peaked at JD 2450197 which is about 37 days after the hard X-ray detection by SIGMA-Granat and about 31 days after the soft X-ray peak. As discussed by Hjellming et al. (1997) the fluctuating radio emission seen between JD 2450250 and JD 2450336 seems to roughly follow the "glitches" in the 2-12 keV RXTE ASM light curve that peak at about JD $2450190,2450240,2450290$, and 2450310 . In the data for five radio frequencies a radio glitch also occurs during the decay of the first event at JD 2450204. If one then associates the X-ray "peaks" with radio "peaks" at JD 2450197 , $2450204,2450260,2450294$, and 2450330 the XR-radio delays are roughly 31 , 


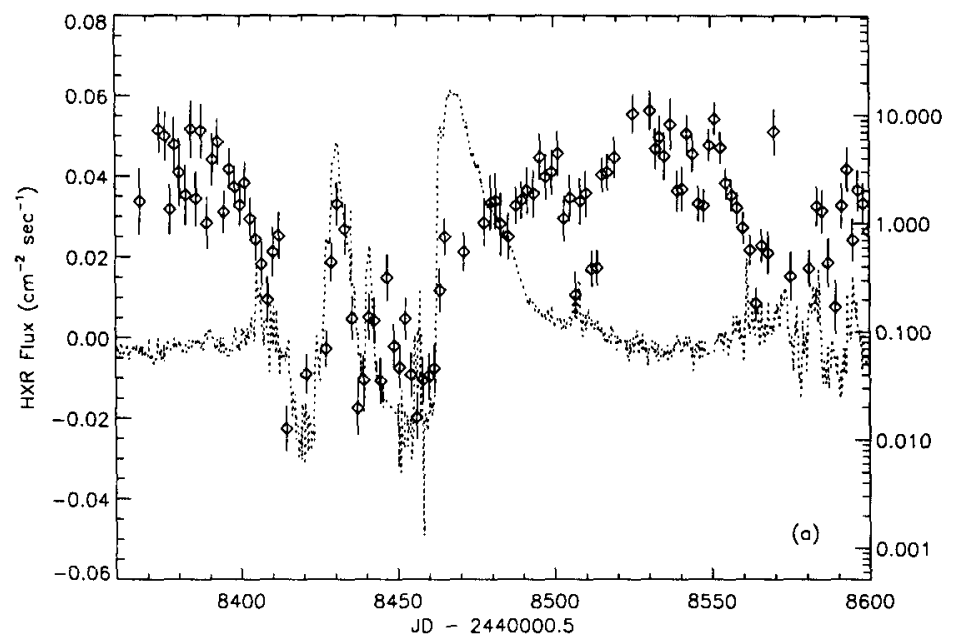

Figure 6. BATSE 20-100 keV fluxes (left ordinate axis, open circles) and $2.7 \mathrm{GHz}$ radio fluxes (right axis, dotted line) are plotted as a function of Julian Day (JD) -2440000.5 for a Cyg X-3 flaring event in 1991.

$14,20,4$, and 20 days. These delay times are not well-defined because of the fuzzy definition of some X-ray and/or radio "peaks", but are indicative of radio enhancements occurring about 35 days after the first and major peak. The delays are of the order of 15 days for later, weaker glitches. These are qualitatively similar to the delays for the three 1994 XR-radio events in GRO J1655-40, but seem to be significantly longer.

As discussed by Hjellming et al. (1997) the radio spectrum for GRS 1739278 was optically thin with a spectral index of -0.6 for both the rise of the radio light curve and during the decay of the initial event. This is strong evidence that the radio emission for this event was in the form of optically thin jets, as imaged in GRO J1655-40, since a symmetrically expanding synchrotron source cannot avoid being optically thick before the peak in a flare event (van der Laan 1966, Hjellming and Johnston 1988).

\section{X-ray-Radio Correlations In Cyg X-3}

The delayed correlation between hard X-ray flares, as seen by BATSE, and the ejection of relativistic radio jet material as seen in either images or radio light curves, prompted a detailed comparison (McCullough et al., 1997) between the BATSE and Green Bank Interferometer (GBI) radio light curves for the galactic $\mathrm{X}$-ray binary Cyg X-3 for the nearly five year period for which they had data in common. Whenever there was flaring and pre-flaring activity, the hard Xray flux is correlated with the radio flux, particularly during the pre-flares dips which are well-known in the radio data for $\mathrm{Cyg} X-3$, but which were seen for 
the first time in the 20-100 keV BATSE fluxes. In addition, whenever the radio flux was quiescent and only slowly varying, there is a specific anti-correlation between a high, steady level of hard X-ray flux and radio flux. In Figure 6 these correlations and anti-correlations are shown for a 240 day period in 1991. The correlations during pre-flare activity are similar to those in the delayed XRradio events for the $\mathrm{X}$-ray transients discussed in this paper in the sense that an initial hard X-ray state is followed by an interlude of low hard X-ray and radio emission, and some tens of days later a large radio flare begins - and only during the decay of the radio flare does the X-ray source return to its previous hard state. The pre-flare hard X-ray and radio dips probably identify the beginnings of small, but expanding, optically thick radio jets. On the occasion of several major flares Cyg X-3 radio emission has been observed to have expansion in the North-South direction with velocities of the order of $0.3 \mathrm{c}$, which is consistent with this scenario (Schalinski et al. 1995).

\section{Summary}

What does it mean when one sees synchrotron radio emission from X-ray binaries and transients? Primarily that relativistic electrons with $100-1000 \mathrm{MeV}$ energy are present, and mixed with magnetic fields, and that these electrons must have been accelerated to these energies outside the high radiation density inside the binary system. When relativistic bulk motions (0.26-0.92c) are seen (SS433, Cyg X-3, GRS1915+105, GRO J1655-40), this is a result of outflows from the accretion disk environment. Other papers in this conference indicate that this outflow results from winds, mixed with magnetic fields, from rotating accretion disks, which collimate as jets on the axis of the accretion disk at large distances. The jet velocities are proportional to the rotational velocity at the inner accretion disk (Blandford and Payne 1982).

Table 1. Relative Timing of X-ray and Radio Events

\begin{tabular}{lllllllll}
\hline Name & Event & $\begin{array}{l}\text { X-ray } \\
\text { Start }\end{array}$ & $\begin{array}{l}\text { X-ray } \\
\text { Peak }\end{array}$ & $\begin{array}{l}\text { X-ray } \\
\text { End }\end{array}$ & $\begin{array}{l}\text { Radio } \\
\text { Peak }\end{array}$ & $\begin{array}{l}\text { XR- } \\
\text { Radio } \\
\text { Delay } \\
\text { [day] }\end{array}$ & $\begin{array}{l}\text { Jet } \\
\text { Start } \\
\text { [day] }\end{array}$ & $\begin{array}{l}\text { Jet } \\
\text { Delay } \\
\text { [day] }\end{array}$ \\
\hline GRO J1655-40 & B1 & 9561 & 9569 & 9581 & 9578 & $\sim 13$ & 9574 & $\sim 5$ \\
1994 Events & B2 & 9600 & 9607 & 9612 & 9608 & $\sim 2$ & 9605 & $\sim-2$ \\
& B3 & 9655 & 9662 & & 9671 & $\sim 1$ & 9668 & $\sim 6$ \\
GRO J1655-40 & X1 & 0206 & 0212 & & $0223-231$ & $11-19$ & \\
1996 Event & X2 & 0225 & & & & & \\
& B1 & 0230 & & & $0223-231$ & $\sim 0$ & \\
GRS 1716-249 & B1 & 9700 & 9763 & 9770 & $<9775$ & $?-13$ & \\
& B3,B4 & & & & & & \\
& B5 & 9890 & 9940 & 9950 & 9949 & $9-12$ & \\
GRS 1739-278 & X1 & 0120 & 0166 & & -9952 & & \\
& & -140 & & & & $\sim 31$ & \\
& X2 & 0188 & 0190 & & 0204 & $\sim 14$ & \\
& X3 & 0220 & 0240 & & 0260 & $\sim 20$ & \\
& X4 & 0220 & 0290 & & 0294 & $\sim 4$ & \\
X5 & 0220 & 0310 & & 0330 & $\sim 20$ & \\
\hline \hline
\end{tabular}


The X-ray-radio connection discussed in this paper for the events in GRO J1655-40, GRS 1716-249, and GRS 1719-278 provide the first data we have on how radio events couple to $X$-ray events. In Table 1 we list the events in these objects (with Xn and Bn identifying soft and hard X-ray events) their beginning, peaks, and end - together with how the $\mathrm{X}$-ray peaks relate to radio peaks and jet ejection. It is most noteworthy that for the 1994 events in GRO J1655-40 there are 1-13 day delays between hard $\mathrm{X}$-ray and radio peaks, but in this case, where the VLBA images of jet evolution identify the times jet ejection began, these beginnings are all within a few days of the hard X-ray peaks. As seen in Table 1 , there is some indication that the delay between hard X-ray and radio peaks is the order of $\sim 15$ days for the first and biggest outburst events, and becomes short for weaker, later radio events. The longer delays for GRS 1739-278, which occur with respect to soft X-ray peaks, may be consistently longer than delays after hard X-ray peaks.

\section{Conclusions}

The high resolution imaging of the superluminal radio jets in GRO J1655-40, together with the hard $\mathrm{X}$-ray and radio light curves associated with the starting and stopping of the ejection of these jets, indicates a new paradigm for the relationship of radio events following hard X-ray events. This paradigm seems to also be followed in the hard X-ray-radio relationship before and during strong radio flaring in Cyg $X-3$, which is a know relativistic jet source during flaring. The paradigm is that a hard $\mathrm{X}$-ray state peaks, or terminates, at the time radio jet material radiating by synchrotron emission begins its outward motion from the central binary system. Since this radio emission decays due to adiabatic energy losses, the peak in the light curve occurs some tens of days later when jet ejection has stopped while the decay of radio surface brightness continues, or in the case of Cyg X-3, when the jets change from optically thick to optically thin. For the X-ray transients the jet emission mostly seems to be optically thin. In the case of GRS 1739-278 the optically thin rise of the radio light curve is clearly seen.

Thus only two years after the new relativistic jet paradigm was directly observed in GRS 1655-40 there have been further events in GRO J1655-40 and GRS 1716-249, and a new transient GRS 1739-278. These events provide indirect light curve evidence for the paradigm of a hard-soft X-ray state transition followed immediately by relativistic jet ejection with radio light curves peaking some tens of days after the change of X-ray state.

Acknowledgments. The National Radio Astronomy Observatory is operated by Associated Universities, Inc., under a cooperative agreement with the U.S. National Science Foundation.

\section{References}

Bailyn, C.D. et al. 1995a, Nature, 374, 701

Bailyn, C.D. et al. 1995b, Nature, 378, 157

Ballet, J., Denis, M., Gilfanov, M., \& Sunyaev, R. 1993, IAUC Circ. 5874 
Blandford, R.D. and Payne, D.G. 1982, MNRAS, 199, 883

Borozdin, K., Alexandrovich, N., \& Sunyaev, R. 1995, IAUC Circ. 6350

Harmon, B.A. et al. 1993, IAU Circ. 5834

Harmon, B.A. et al. 1995, Nature, 374, 703

Hjellming, R.M. \& Han, X.-H. 1995, In X-ray Binaries (Cambridge: London), Ed. Lewin et al. , p. 308

Hjellming, R.M. \& Johnston, K.J. 1988, ApJ, 328, 600

Hjellming, R.M. \& Rupen, M.P. 1995, Nature, 375, 464

Hjellming, R.M. et al. 1996, ApJ Letters, Oct. 20 issue

Hjellming, R.M., Rupen, M.P., Dhawan, V., Remillard, R., Marti, J, Mirabel, I.F., \& Rodriguez, L.F. 1997, ApJ, in preparation.

McCullough; M. et al. 1997, ApJ Letters, in preparation.

Paul, J., Bouchet, L., Churasov, E., \& Sunyaev, R. 1996, IAU Circ. 6348

Schalinski, C.J. et al. 1995, ApJ, 447, 752-759

Tavani, M. et al. 1996, ApJ Letters, in press

Tingay, J. et al. 1995, Nature, 374, 141

van der Laan, H. 1966, Nature, 211, 1131. 